\title{
Non circular Unitary ESPRIT-MUSIC for angle estimation in bistatic MIMO radar
}

\author{
Wei Wang ${ }^{\mathrm{a})}$, Xianpeng Wang, Xin Li, and Moxuan Li \\ College of Automation, Harbin Engineering University, Harbin, 150001, Heilongjiang \\ P.R, China \\ a)chinaww2006@yahoo.com.cn
}

Abstract: In this letter, the bistatic Multiple-Input Multiple-Output radar with non circular signal is considered, and a novel non circular Unitary ESPRIT-MUSIC algorithm for the joint direction of departure (DOD) and direction of arrival (DOA) is presented. Firstly, the properties of the non circular signals are utilized to extend the received data, which corresponds to double elements of MIMO virtual array. Then the complex valued data matrix can be converted into a real valued matrix by Unitary transformation, and the DODs and DOAs can be estimated by Unitary ESPRIT and Unitary Root-MUSIC, respectively. The proposed method can provide better angle estimation performance and handle more targets than Unitary ESPRIT-MUSIC. Some simulation results are used to show the performance of the proposed method.

Keywords: MIMO radar, non circular signal, Unitary

ESPRIT-MUSIC, angle estimation

Classification: Antennas and Propagation

\section{References}

[1] L. Jian and P. Stoica, "MIMO radar with colocated antennas," IEEE Signal Process. Mag., vol. 24, no. 5, pp. 106-114, 2007.

[2] M. L. Bencheikh and L. Wang, "Joint DOD-DOA estimation using combined ESPRIT-MUSIC approach in MIMO radar," Electron. Lett., vol. 46, no. 15, pp. 1081-1083, 2010.

[3] W. Wei, W. Xianpeng, L. Moxuan, and L. Xin, "Angle estimation using combined Unitary ESPRIT-MUSIC algorithm for MIMOradar," IEICE Commun. Express, vol. 1, no. 4, pp. 154-159, 2012.

[4] M. Haard and J. A. Nossek, "Unitary ESPRIT: how to obtain increased estimation accuracy with reduced computational burden," IEEE Trans. Signal Process., vol. 43, no. 5, pp. 1232-1242, 1995.

[5] M. Pesavento, A. B. Gershman, and M. Haaedt, "Unitary root-MUSIC with a real-valued eigendecomposition: A theoretical and experimental performance study," IEEE Trans. Signal Process., vol. 48, no. 5, pp. 13061314, 2000.

[6] C. Pascal, W. Yide, and S. Joseph, "A non-circular sources direction finding method using polynomial rooting," Signal Processing, no. 81, pp. 1765$1770,2001$. 


\section{Introduction}

Multiple-input multiple-output (MIMO) radar, which utilizes multiple antennas to simultaneously transmit orthogonal waveforms at the transmit side and receive the reflected signal at the receive side, has drawn considerable attention in recent years [1]. Joint the direction of departure (DOD) and the direction of arrival (DOA) estimation is very improtant aspect in bistatic MIMO radar. In order to solve this problem, a combined ESPRIT-MUSIC method is proposed in [2], which exploits the invariance property of the transmit array to estimate DOD and the Root-MUSIC technology to determine the DOA. In [3], a combined Unitary ESPRIT-MUSIC algorithm is presented to angle estimation. The DOD and DOA are estimated by Unitary ESPRIT and Unitary Root-MUSIC, respectively. The Unitary ESPRIT-MUSIC method provides lower computational complexity and slightly better angle estimation performance than ESPRIT-MUSIC. But the above mentioned methods do not consider the properties of non circular signals. In this letter, the non circular signals are used in bistatic MIMO radar and a novel non circular Unitary ESPRIT-MUSIC (NC Unitary ESPRIT-MUSIC) algorithm for the joint DOD and DOA estimation is proposed, which has following advantages: (1) owing to exploit the properties of the non circular singal, it provides better angle estimation performance and detects more targets than Unitary ESPRIT-MUSIC; (2) the DODs and DOAs are automatically paired.

\section{Problem formulation}

Consider a narrowband bistatic MIMO radar system with $M$ closely spaced transmit antennas and $N$ closely spaced receive antennas, both of them are half-wavelength spaced uniform linear arrays and all the elements are omnidirectional. At the transmit side, $M$ orthogonal noncircular signals supposed BPSK modulated are transmitted simultaneously. At the receive side, the echoed signals are processed by all the transmitted waveforms. It is assumed that the number of targets is known, and there are $P$ uncorrelated targets located at the same range bin. The pth target is lacated at $\left(\varphi_{p}, \theta_{p}\right)$, where $\varphi_{p}$ denotes the direction of the $p t h$ target with respect to the transmit array (i.e. DOD) and $\theta_{p}$ denotes the direction of the $p t h$ target with respect to the receive array (i.e. DOA). Then the output of all the matched filters at the receiver can be written as

$$
\mathbf{x}(t)=\mathbf{A} \boldsymbol{\Delta} \mathbf{s}(t)+\mathbf{n}(t)
$$

where $\mathbf{A}=\left[\mathbf{a}\left(\varphi_{1}, \theta_{1}\right), \mathbf{a}\left(\varphi_{2}, \theta_{2}\right), \ldots, \mathbf{a}\left(\varphi_{P}, \theta_{P}\right)\right]$ is an $M N \times P$ transmit-receive steering matrix, $\mathbf{a}\left(\varphi_{p}, \theta_{p}\right)=\mathbf{a}\left(\varphi_{p}\right) \otimes \mathbf{a}\left(\theta_{p}\right)(p=1,2, \cdots, P)$ is the transmitreceive steering vector of the $p$ th target, $\otimes$ denotes the Kronecker product. $\mathbf{a}(\theta)=[1, \exp (j \pi \sin \theta), \ldots, \exp (j \pi(N-1) \sin \theta)]^{\mathrm{T}}$ and $\mathbf{a}(\varphi)=[1, \exp (j \pi \sin \varphi)$, $\ldots, \exp (j \pi(M-1) \sin \varphi)]^{\mathrm{T}}$ are the receive steering vector and the transmit steering vector, respectively. $\mathbf{s}(t)=\left[s_{1}(t), s_{2}(t) \ldots, s_{P}(t)\right]^{\mathbf{T}}$ is a noncircular signal supposed BPSK modulated, which is satisfied with $\mathbf{s}(t)=\mathbf{s}^{*}(t)$, where $(\cdot)^{*}$ denotes the complex conjugation without transposition. $\boldsymbol{\Delta}=$ 
$\operatorname{diag}\left\{e^{j \psi_{1}}, \ldots, e^{j \psi_{P}}\right\}$ denotes the arbitrary phase of the reflected signals. $\mathbf{n}(t)$ is an $M N \times 1$ complex Gaussian white noise vector with zeros means and covariance matrix $\sigma^{2} \mathbf{I}_{M N}$, where $\mathbf{I}_{K}$ denotes a $K \times K$ identify matrix.

\section{Non circular Unitary ESPRIT-MUSIC for angle estimation}

In order to exploit the non circularity of signals, the received data can be extended as following

$$
\mathbf{Y}(t)=\left[\begin{array}{c}
\mathbf{x}(t) \\
\boldsymbol{\Gamma}_{M N} \mathbf{x}^{*}(t)
\end{array}\right]=\left[\begin{array}{c}
\mathbf{A} \boldsymbol{\Delta} \\
\boldsymbol{\Gamma}_{M N} \mathbf{A}^{*} \boldsymbol{\Delta}^{*}
\end{array}\right] \mathbf{s}(t)+\left[\begin{array}{c}
\mathbf{n}(t) \\
\boldsymbol{\Gamma}_{M N} \mathbf{n}^{*}(t)
\end{array}\right]
$$

where $\boldsymbol{\Gamma}_{k}$ denotes the $K \times K$ exchange matrix with ones on its anti-diagonal and zeros elsewhere. The extended received data allows to increase the observation space while keeping the dimension of the signal subspace unchanged. The extended transmit-receive steering vector is then

$$
\mathbf{b}\left(\varphi_{p}, \theta_{p}\right)=\left[\begin{array}{c}
a\left(\varphi_{p}, \theta_{p}\right) e^{j \psi_{p}} \\
\boldsymbol{\Gamma}_{M N}\left(a\left(\varphi_{p}, \theta_{p}\right)\left(e^{j \psi_{p}}\right)\right)^{*}
\end{array}\right]
$$

Then the extended transmit-receive steering matrix can be written as $\mathbf{B}=\left[\mathbf{b}\left(\varphi_{1}, \theta_{1}\right), \mathbf{b}\left(\varphi_{2}, \theta_{2}\right), \ldots, \mathbf{b}\left(\varphi_{P}, \theta_{P}\right)\right]$. The number of virtual elements with respect to the extended transmit-receive steering vector is twice as much as the MIMO vitual array. Assume that the number of snapshots is $L$, and the received data matrix can be formulated as $\overline{\mathbf{Y}}=\left[\mathbf{Y}\left(t_{1}\right), \mathbf{Y}\left(t_{2}\right), \ldots, \mathbf{Y}\left(t_{L}\right)\right]$. Then the received data matrix can be extended as $\mathbf{Z}=\left[\begin{array}{ll}\overline{\mathbf{Y}} & \boldsymbol{\Gamma}_{2 M N} \overline{\mathbf{Y}}^{*} \boldsymbol{\Gamma}_{L}\end{array}\right]$. In [4], it has been proved that the extended data matrix $\mathbf{Z}$ is a centro-Hermitian matrix. Thus, the extended data matrix $\mathbf{Z}$ can be converted into a real valued matrix by using unitary transformation, which is expressed as

$$
\mathbf{Z}_{\text {real }}=\mathbf{U}_{2 M N}^{\mathrm{H}}\left[\begin{array}{ll}
\mathbf{Z} & \boldsymbol{\Gamma}_{2 M N} \mathbf{Z}^{*} \boldsymbol{\Gamma}_{L}
\end{array}\right] \mathbf{U}_{2 L}
$$

Where $(\cdot)^{\mathrm{H}}$ denotes the Hermitian transpose, $\mathbf{U}_{K}$ is a unitary matrix which is defined as

$$
\mathbf{U}_{2 K}=\frac{1}{\sqrt{2}}\left[\begin{array}{cc}
\mathbf{I}_{K} & j \mathbf{I}_{K} \\
\boldsymbol{\Gamma}_{K} & -j \boldsymbol{\Gamma}_{K}
\end{array}\right], \mathbf{U}_{2 K+1}=\frac{1}{\sqrt{2}}\left[\begin{array}{ccc}
\mathbf{I}_{K} & 0 & j \mathbf{I}_{K} \\
\mathbf{0}^{\mathrm{T}} & \sqrt{2} & \mathbf{0}^{T} \\
\boldsymbol{\Gamma}_{K} & 0 & -j \boldsymbol{\Gamma}_{K}
\end{array}\right]
$$

Using the ML estimation, the real valued covariance matrix of $\mathbf{Z}_{\text {real }}$ for $2 L$ snapshots can be expressed as $\mathbf{R}_{\text {real }}=1 /(2 L) \mathbf{Z}_{\text {real }} \mathbf{Z}_{\text {real }}^{H}$. Then the signal subspace $\mathbf{E}_{s}$ and the noise subspce $\mathbf{E}_{n}$ can be obtained by the eigendecomposition of $\mathbf{R}_{\text {real }}$, respectively, where $\mathbf{E}_{s}$ is the $2 M N \times P$ matrix which is the eigenvectors corresponding to the $P$ largest eigenvalues of $\mathbf{R}_{\text {real }}$, and $\mathbf{E}_{n}$ is the $2 M N \times(2 M N-P)$ matrix which is the eigenvectors corresponding to the smallest eigenvalues of $\mathbf{R}_{\text {real }}$. After the unitary transformation, the extended real valued transmit-receive steering vector can be written as $\mathbf{g}\left(\varphi_{p}, \theta_{p}\right)=\mathbf{U}_{2 M N}^{\mathrm{H}} \mathbf{b}\left(\varphi_{p}, \theta_{p}\right),(1 \leq p \leq P)$ [4]. Then the extended real valued transmit-receive steering matrix can be defined as $\mathbf{G}=$ $\left[\mathbf{g}\left(\varphi_{1}, \theta_{1}\right), \mathbf{g}\left(\varphi_{2}, \theta_{2}\right), \ldots, \mathbf{g}\left(\varphi_{P}, \theta_{P}\right)\right]$. According to the literature [5], the real 
valued noise subspace is orthogonal to the extended real-valued transmitreceive steering vector, i.e. $\left\|\mathbf{E}_{n}^{\mathrm{H}}\left(\mathbf{U}_{2 M N}^{\mathrm{H}} \mathbf{g}(\varphi, \theta)\right)\right\|^{2}$ which can be written as

$$
\mathbf{f}^{\mathrm{H}}(\theta) \mathbf{f}^{\mathrm{H}}(\varphi) \mathbf{U}_{2 M N} \mathbf{E}_{n} \mathbf{E}_{n}^{\mathrm{H}} \mathbf{U}_{2 M N}^{\mathrm{H}} \mathbf{f}(\varphi) \mathbf{f}(\theta)=0
$$

where

$$
\mathbf{f}(\theta)=\left[\begin{array}{c}
\mathbf{a}(\theta) e^{j \psi} \\
\mathbf{a}^{*}(\theta)\left(e^{j \psi}\right)^{*}
\end{array}\right], \quad \mathbf{f}(\varphi)=\left[\begin{array}{cc}
\mathbf{a}(\varphi) \otimes \mathbf{I}_{N} & \mathbf{0} \\
\mathbf{0} & \boldsymbol{\Gamma}_{M N} *\left(\mathbf{a}^{*}(\varphi) \otimes \mathbf{I}_{N}\right)
\end{array}\right]
$$

From the Eq. (6) and (7), it can be observed that the DOD and DOA of target can be estimated separately in real valued space. Therefore, the proposed method can be subdivided in two steps. In the first step, the DOD can be estimated by Unitary ESPRIT. In the second step, for each estimated DOD, the corresponding steering matrix $\mathbf{f}(\varphi)$ is substituted in Eq. (6), then the DOA can be estimated by Unitary Root-MUSIC.

Firstly, we determine the DOD by Unitary ESPRIT. Let $\mathbf{B}_{t 1}=\boldsymbol{\Pi}_{1} \mathbf{B}$ and $\mathbf{B}_{t 2}=\boldsymbol{\Pi}_{2} \mathbf{B}$, where $\boldsymbol{\Pi}_{1}=\left[\begin{array}{ll}1 & 0 \\ 0 & 1\end{array}\right] \otimes \mathbf{J}_{1}$ and $\boldsymbol{\Pi}_{2}=\left[\begin{array}{ll}1 & 0 \\ 0 & 1\end{array}\right] \otimes \mathbf{J}_{2}$ are $2(M N-$ $N) \times 2 M N$ selection matrices, respectively, $\mathbf{J}_{1}=\left[\begin{array}{ll}\mathbf{I}_{N(M-1)} & \mathbf{0}_{N(M-1) \times N}\end{array}\right]$ and $\mathbf{J}_{2}=\left[\begin{array}{ll}\mathbf{0}_{N(M-1) \times N} & \mathbf{I}_{N(M-1)}\end{array}\right]$. According to the configuration of the extended transmit-receive steering vector in Eq. (3), there exists a complex valued shift invariance relation between $\mathbf{B}_{t 1}$ and $\mathbf{B}_{t 2}$, which can be written as $\mathbf{B}_{t 2}=\mathbf{B}_{t 1} \boldsymbol{\Psi}_{t}$, where $\boldsymbol{\Psi}_{t}$ is a diagonal matrix with diagonal elements $\gamma_{p}=\exp \left(j \pi \sin \varphi_{p}\right)$ for $p=1,2, \ldots, P$. Owing to the centrosymmetric array configuration of the extended transmit-receive steering vector, the complexvalued shift invariance relation can be turned into the real-valued shift invariance relation [4]

$$
\mathbf{D}_{2} \mathbf{G}=\mathbf{D}_{1} \mathbf{G} \boldsymbol{\Phi}_{t}
$$

where $\mathbf{D}_{1}=\operatorname{Re}\left\{\mathbf{U}_{2 N(M-1)} \boldsymbol{\Pi}_{2} \mathbf{U}_{2 M N}\right\}$ and $\quad \mathbf{D}_{2}=\operatorname{Im}\left\{\mathbf{U}_{2 N(M-1)} \boldsymbol{\Pi}_{2} \mathbf{U}_{2 M N}\right\}$ are real-valued matrices, $\boldsymbol{\Psi}_{t}$ is real-valued diagonal matrix with diagonal elements $\lambda_{p}=\tan \left(\pi \sin \varphi_{p} / 2\right)$ for $p=1,2, \ldots, P$, which represents the realvalued shift invariance relation. The relationship between the real valued transmit-receive steering matrix $\mathbf{G}$ and the real valued signal subspace $\mathbf{E}_{s}$ can be expressed as $\mathbf{E}_{s}=\mathbf{G T}$, where $\mathbf{T}$ is a nonsingular matrix. Then substituting $\mathbf{E}_{s}=\mathbf{G T}$ into Eq. (8), we can obtain

$$
\mathbf{D}_{2} \mathbf{E}_{s}=\mathbf{D}_{1} \mathbf{E}_{s} \boldsymbol{\Theta}_{t}
$$

where $\boldsymbol{\Theta}_{t}=\mathbf{T}^{-1} \boldsymbol{\Phi}_{t} \mathbf{T}$. The Eq. (9) can be solved by the least squares (LS) or the total least squares (TLS), and the rotational invariance matrix $\boldsymbol{\Theta}_{t}$ can be obtained. Then the diagonal matrix $\boldsymbol{\Phi}_{t}$ can be obtained by eigendecomposition of $\boldsymbol{\Theta}_{t}$, and the DOD can be estimated as $\hat{\varphi}_{p}=\arcsin \left(2 \arctan \left(\lambda_{p}\right) / \pi\right)$. For each estimated DOD, the estimated transmit steering vector can be written as

$$
\mathbf{a}\left(\hat{\varphi}_{p}\right)=\left[1, \exp \left(j \pi \sin \hat{\varphi}_{p}\right), \ldots, \exp \left(j \pi(M-1) \sin \hat{\varphi}_{p}\right)\right]^{\mathrm{T}}
$$

Then substituting Eq. (10) into Eq. (6), we have

$$
\mathbf{d}^{\mathrm{H}} \mathbf{C}^{\mathrm{T}}\left(1 / z_{r}\right)\left[\begin{array}{ll}
\mathbf{E}_{n 11} & \mathbf{E}_{n 12} \\
\mathbf{E}_{n 21} & \mathbf{E}_{n 22}
\end{array}\right] \mathbf{C}\left(z_{r}\right) \mathbf{d}=0
$$


where

$$
\begin{gathered}
\mathbf{d}=\left[\begin{array}{c}
e^{j \phi} \\
e^{-j \phi}
\end{array}\right], \quad \mathbf{C}\left(z_{r}\right)=\left[\begin{array}{cc}
\mathbf{a}\left(z_{r}\right) & \mathbf{0} \\
\mathbf{0} & \mathbf{a}^{*}\left(z_{r}\right)
\end{array}\right], \quad z_{r}=\exp (j \pi \sin \theta) \\
\mathbf{a}\left(z_{r}\right)=\left[1, z_{r}, \ldots, z^{N-1}\right]^{\mathrm{T}},\left[\begin{array}{ll}
\mathbf{E}_{n 11} & \mathbf{E}_{n 12} \\
\mathbf{E}_{n 21} & \mathbf{E}_{n 22}
\end{array}\right]=\mathbf{f}^{\mathrm{H}}(\hat{\varphi}) \mathbf{U}_{2 M N} \mathbf{E}_{n} \mathbf{E}_{n}^{\mathrm{H}} \mathbf{U}_{2 M N}^{\mathrm{H}} \mathbf{f}(\hat{\varphi})
\end{gathered}
$$

where $\mathbf{E}_{n i j},(i, j=1,2)$ is $M N \times M N$ block matrix. The Eq. (11) can be solved by finding the roots of the following ploynomial

$$
\operatorname{det}\left[\mathbf{C}^{\mathrm{T}}\left(1 / z_{r}\right)\left[\begin{array}{ll}
\mathbf{E}_{n 11} & \mathbf{E}_{n 12} \\
\mathbf{E}_{n 21} & \mathbf{E}_{n 22}
\end{array}\right] \mathbf{C}\left(z_{r}\right)\right]=0
$$

The Eq. (14) can be expressed as [6]

$$
\sum_{i=1}^{4 N-3} q_{i} z_{r}^{i-1-2(N-1)}=0
$$

The paremeters $q_{i}$ in Eq. (15) can be caculated as given in [6]. Thus, for the DOD of the pth target, the corresponding DOA is equivalent to find the root $\hat{z}_{r p}$ inside and closest to the unitary circle of the Eq. (15). Then the DOA of the $p$ th target can be derived as $\hat{\theta}_{p}=\arcsin \left(\angle\left(\hat{z}_{r p}\right) / \pi\right)$, where $\angle(r)$ denotes the phase of $r$. We note that the DOD and DOA can be paired automatically, which avoids the additional pairing procedure. Up to now, we have proposed the non circular Unitary ESRPIT-MUSIC method for angle estimation in bistatic MIMO rdar. The same as to Unitary ESPRIT-MUSIC, the proposed method only can be used for the bistatic MIMO radar with uniform linear array. The proposed method exploits the non circular signals to enlarge the virtual array of bistatic MIMO radar, so it can provide better angle estimation performance and handle more targets than the classical techiques. The maximum number of targets that can be identified by the proposed method is $2 N(M-1)$.

\section{Simulation results}

Assume that the number of targets is known, and there exist $P$ noncoherent targets. The number of snapshots is $L=200$ for an $M=4$ and $N=6$ bistatic MIMO radar.

Figure 1 shows the variation of the RMSEs of the proposed method and the method in $[2,3]$ with SNR, where $P=3$ targets are lacated at angles $\left(\varphi_{1}, \theta_{1}\right)=\left(10^{\circ},-10^{\circ}\right),\left(\varphi_{2}, \theta_{2}\right)=\left(-8^{\circ}, 0^{\circ}\right),\left(\varphi_{3}, \theta_{3}\right)=\left(0^{\circ}, 8^{\circ}\right)$. It is showed that the proposed method has better angle estimation performance than both ESPRIT-MUSIC and Unitary ESPRIT-MUSIC, especially for the low SNR case. Figure 2 shows the variation of the RMSEs with the angle separation, where $P=2$ targets are located at angles $\left(\varphi_{1}, \theta_{1}\right)=\left(0^{\circ},-1^{\circ}\right),\left(\varphi_{2}, \theta_{2}\right)=$ $\left(0^{\circ}+\nabla,-1^{\circ}+\nabla\right), \nabla$ is varied from $1^{\circ}$ to $11^{\circ}$. The SNRs of two targets are $10 \mathrm{~dB}$. From the Fig. 2, it can be seen that the angle estimation accuracy can be improved with the increasing angular separation. When the two 


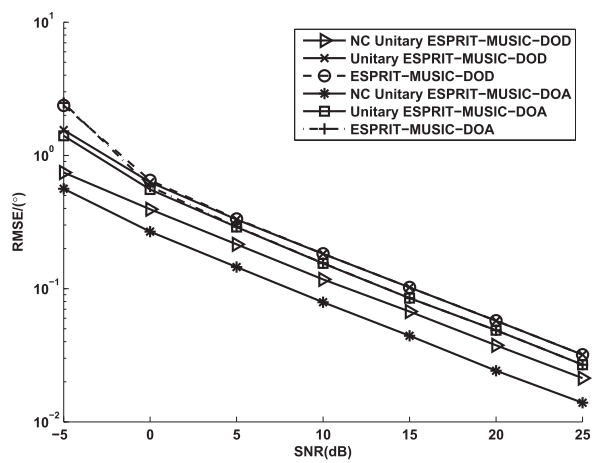

Fig. 1. RMSE of angle estimation against SNR

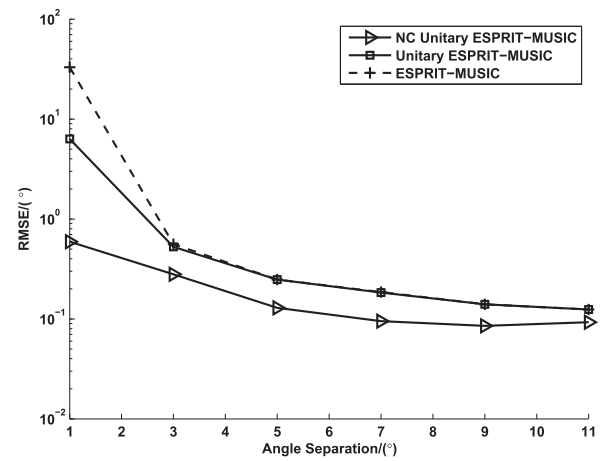

Fig. 2. RMSE of angle estimation versus angle separation

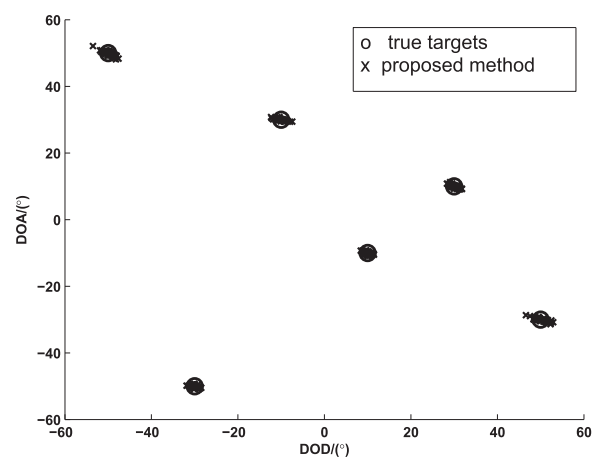

Fig. 3. Angle estimation results for $P=6$ targets with $M=2$ and $N=3$

targets are too close, the ESPRIT-MUSIC and Unitary ESPRIT-MUSIC fail in separating the two targets, while the proposed method also can perform correctly. Figure 3 shows the angle estimation results for $P=6$ targets with $M=2$ and $N=3$, where the lacations of targets are plotted in Fig. 3 and the SNRs of 6 targets are $10 \mathrm{~dB}$. From the Fig. 3, we can observe that $P=6$ targets are paired automatically and correctly by the proposed method. Thus, the proposed method can detect 6 targets with $M=2$ and $N=3$, while the ESPRIT-MUSIC and Unitary ESPRIT-MUSIC are limited to 3 targets. 


\section{Conclusion}

Considering the bistatic MIMO radar with non circular signals, we have proposed a novel non circular Unitary ESPRIT-MUSIC algorithm for joint DOD and DOA estimation. This technique exploits the properties of non circular signals to extend the received data, which corresponds to a larger virtual array. Then the Unitary ESPRIT and Unitary Root-MUSIC method is applied to estimate DOD and DOA, respectively. Simulation results verify that the proposed method has better angle estimation performance and detects more targets than ESPRIT-MUSIC and Unitary ESPRIT-MUSIC.

\section{Acknowledgments}

This work was supported by the Program for New Century Excellent Talents in University (NCET-11-0827) and the National Natural Science Foundation of China (60704018). 